\title{
Kudzu and sleeper cells: the varied ecology of respiratory infections
}

\author{
Robert P. Dickson ${ }^{1,2}$ \\ Affiliations: 'Division of Pulmonary and Critical Care Medicine, Dept of Internal Medicine, University of \\ Michigan Medical School, Ann Arbor, MI, USA. ${ }^{2}$ Michigan Center for Integrative Research in Critical Care, Ann \\ Arbor, MI, USA. \\ Correspondence: Robert P. Dickson, Pulmonary and Critical Care Medicine, University of Michigan Health \\ System, 6220 MSRB III/SPC 5642, 1150 W. Medical Center Dr., Ann Arbor, MI 48109-5642, USA. \\ E-mail: rodicksolamed.umich.edu
}

@ERSpublications

We have known for over a century that nontuberculous mycobacterial infections differ microbiologically and immunologically from those of typical bacterial pathogens. We are now learning they differ ecologically as well. http://ow.ly/8sau30lCqHz

Cite this article as: Dickson RP. Kudzu and sleeper cells: the varied ecology of respiratory infections. Eur Respir J 2018; 52: 1801607 [https://doi.org/10.1183/13993003.01607-2018].

If you have spent time driving in the southern USA, you have witnessed the vast green scourge that is kudzu. Innocently introduced to America from Japan at the 1876 Centennial Exposition, the kudzu vine has infested the American south, blanketing roadsides and smothering competing vegetation (figure 1). Kudzu can grow a foot $(\sim 30 \mathrm{~cm})$ a day, scales trees and telephone poles, and creates a dense shade that deprives everything beneath it of sunlight. American Southerners insist that when it is quiet in early summer, one can hear the kudzu growing.

Kudzu's strategy (grow fast, get big and crowd out competitors) serves as a decent metaphor for our current ecological understanding of acute bacterial pneumonia. Compared to the diverse but low-abundance bacterial communities detected in healthy lungs (figure 2a), lung microbiota in acute pneumonia resemble a kudzu-overrun roadside: high biomass (measured by total bacterial burden), low diversity, and community domination by a single, usually cultivable pathogen (e.g. Streptococcus pneumoniae, Pseudomonas aeruginosa or Staphylococcus aureus) [1-3] (figure 2b). The rapid clinical onset of acute bacterial pneumonia also mirrors kudzu's breakneck pace, progressing over hours rather than weeks or months. If one auscultates closely enough, one can almost hear the pneumococci dividing.

In this issue of the European Respiratory Journal, Sulaiman et al. [4] provide us with an important exception to this "kudzu" model of lung ecology in respiratory infections. The authors' focus was nontuberculous mycobacteria (NTM), ubiquitous environmental microbes that represent a common, growing and clinically significant cause of lung disease [5]. The authors used the now-familiar molecular techniques of microbial ecology (16S ribosomal RNA (rRNA) gene amplicon sequencing) to characterise bacterial communities in sputum and bronchoalveolar lavage fluid collected from a large and well-matched cohort of patients with non-cystic fibrosis bronchiectasis. The presence of viable NTM was confirmed via concurrent culture on appropriate media and NTM-infected specimens were compared with matched NTM-negative controls. The authors' thoughtful, well-powered and well-controlled approach provides the field with our best glimpse to date of the lung ecology of mycobacterial respiratory infections.

The study's most provocative findings are its negative ones, as they reveal how ecologically dissimilar NTM infections are from acute bacterial pneumonia (figure 2c). When compared to NTM-negative specimens, 
FIGURE 1 The kudzu vine's "infection" of the southern US highway system resembles acute bacterial pneumonia: high biomass, low diversity and community domination by a single invasive species. Chronic mycobacterial infections, we are learning, employ a more insidious ecological strategy. Photograph by Kerry Britton for the US Forest Service (Washington, DC, USA).

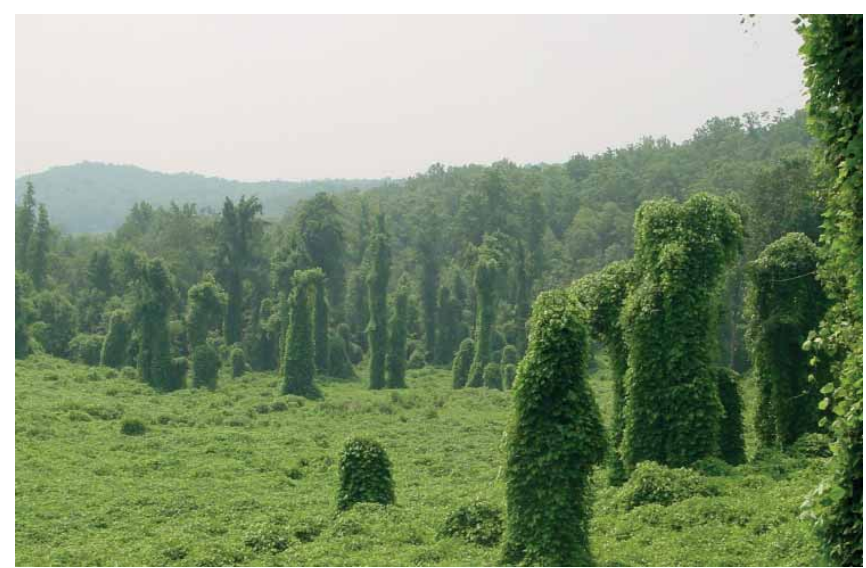

NTM-infected specimens had no detectable increase in total bacterial burden. This differs from acute pneumonia, in which bacterial burden of respiratory specimens is typically 10-1000-fold higher than those of uninfected patients [2, 6]. Similarly, NTM-infected specimens did not differ in their community diversity compared to NTM-negative specimens, failing to resemble the "collapsed community" of acute bacterial pneumonia [2,3]. Finally, whereas in acute bacterial pneumonia, the culture-identified pathogen is usually dominant, representing a wide majority of detected bacteria, the authors found that NTM remains only a minor community member, near or below our molecular limits of detection. By all key ecological indices (biomass, diversity and relative abundance of the pathogen), NTM infections look nothing like the kudzu-like communities of bacterial pneumonia.

A savvy reader may ask if these negative findings are artefactual, reflecting the known technical challenges of studying mycobacteria. The thick, waxy cell wall of mycobacteria is notoriously stubborn against DNA extraction and the genomes of most disease-associated mycobacteria contain only one copy of the amplified 16S rRNA gene [7] (unlike, for example, S. pneumoniae and P. aeruginosa, which have four 16S rRNA genes apiece). Thus NTM are systematically underrepresented in most sequencing-based studies of lung microbiota. The current authors, mindful of these issues, used a complementary approach that was optimised to maximise mycobacterial signal, including a modified DNA extraction protocol [8] and a
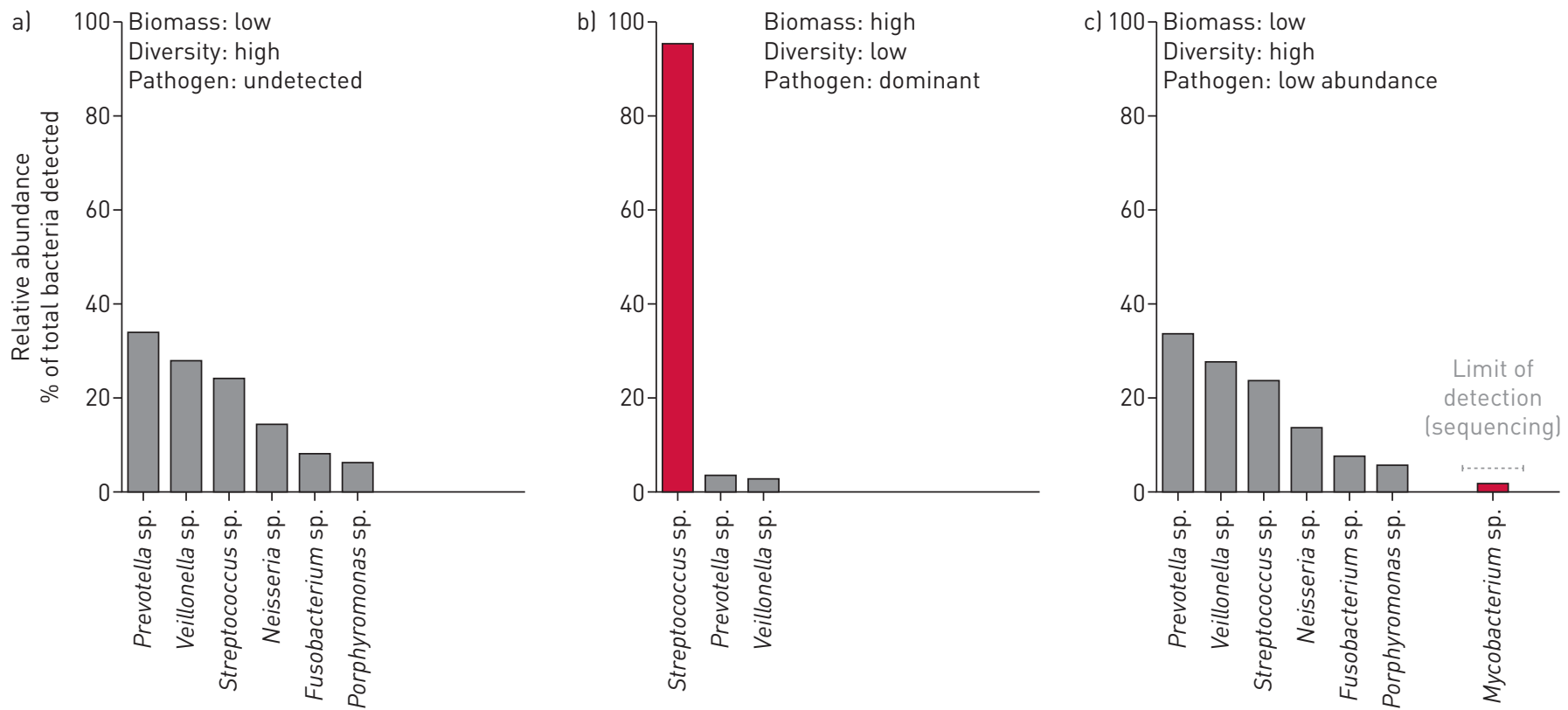

FIGURE 2 Ecological patterns of respiratory infections. a) In health, lung bacterial biomass is low and comprised of a diverse community of oropharynx-associated bacteria. b) In acute bacterial infections, bacterial biomass is high and diversity collapses around a dominant pathogen comprising the vast majority of detected bacteria (the "kudzu" model). c) By contrast, in chronic mycobacterial infections, biomass remains modest, diversity remains unchanged and the pathogen is a minor constituent of the lung community close to the limit of detection for current sequencing technologies. 
nested two-step amplification protocol for enriching sequencing results with mycobacterial reads [9]. Despite this intentional deck-stacking, mycobacteria were still only detected in a minority of NTM-positive specimens ( $47 \%$ of bronchoalveolar lavage specimens and $17 \%$ of sputum specimens). Even with intentionally biased sequencing techniques, the mycobacteria of the infected patients' airways are barely (and not always) detectable.

This important observation that even optimised sequencing techniques cannot detect mycobacteria in most culture-positive specimens should suffice to correct a commonly repeated fallacy: that our modern microbiome tools are more sensitive than conventional cultivation. In fact, culture remains our most sensitive assay for the detection and identification of low-abundance bacteria. Whereas cultivation can, in principle, detect the presence of a single bacterial cell, our sequencing platforms have finite depths of sampling, and are profoundly "noisy" at low bacterial concentrations due to the contaminating influence of environmental and reagent DNA [10,11]. Indeed, when sequencing techniques are complemented by concurrent cultivation, their sensitivity increases considerably $[12,13]$. This common misconception (the superior sensitivity of sequencing) stems from the increased breadth of microbiota reported by sequencing-based approaches, as they provide relative quantification of bacterial taxa independent of their ability to grow in specific selective conditions. The revolution in molecular microbiology has proven invaluable for understanding the complexity of respiratory microbiology but the current study reminds us that it has not improved our ability to detect low-abundance bacteria like NTM.

This observation has key implications for one of the field's most exciting recent developments: the use of real-time metagenomics to rapidly identify respiratory pathogens using direct specimen sequencing. The speed, cost and accessibility of sequencing have all advanced so quickly that what was unthinkable a decade ago has already been shown feasible $[14,15]$ : using direct metagenomic sequencing of patient specimens, respiratory pathogens can be identified with great precision in mere hours, far faster than conventional culture-based protocols. While this approach could prove transformative in accelerating clinical diagnostics and informing antimicrobial stewardship, a key barrier is the high host/microbe ratio of genomic DNA in respiratory specimens [16]. The current study suggests that while real-time sequencing may prove useful in "kudzu" conditions like acute bacterial pneumonia, in which the "signal" of the overgrown pathogen swells to readily detectable levels, the technology is unlikely to replace cultivation for the diagnosis of NTM and smear-negative Mycobacterium tuberculosis infections.

This distinct ecology of NTM infections is compatible with our understanding of the distinct survival strategies used by mycobacterial and typical bacterial pathogens in the hostile microenvironment of the lower respiratory tract. In acute bacterial pneumonia, pathogens thrive via brute force, overwhelming host defences and co-opting the ecological consequences of acute inflammation. In pneumonia, the alveolar space, normally inhospitable to bacterial reproduction [17], is flooded with nutrient-rich oedema [18] providing an otherwise scarce carbon source. The signalling molecules of the host stress response (e.g. tumour necrosis factor (TNF)- $\alpha$, interleukin (IL)-1 $\beta$, IL-6 and catecholamines) selectively promote the growth of bacterial pathogens (e.g. P. aeruginosa, S. aureus and S. pneumoniae) [19-21]. Thus, a self-perpetuating positive feedback loop is established [22]: pathogen growth provokes host inflammation, altering the alveolar microenvironment, which is further exploited by reproducing pathogens. In contrast, mycobacteria use a strategy of stealth, subterfuge and avoidance to persist in the lung. Though pathogenic mycobacteria contain a number of pathogen-associated molecular patterns (PAMPs) capable of provoking brisk immune reactions, they also secrete dedicated surface lipids (e.g. phthiocerol dimycoceroserate) that "mask" their PAMPs, avoiding activation of innate immunity [23]. Mycobacterium avium (the most commonly identified NTM species in the current study) coordinates an exquisite manipulation of alveolar macrophages, stimulating production of anti-inflammatory cytokines (e.g. IL-10), and blunting TNF- $\alpha$ production and antigen presentation [24]. If acute bacterial pathogens use the tactics of "shock and awe", chronic mycobacterial infections are "sleeper cells" content to persist below the limit of detection (both of our assays and of our host defences).

A related and provocative finding of the current study is that the immune tone of the lungs in NTM-infected patients correlates more tightly with their non-mycobacterial lung bacteria than with the detected presence of NTM. Put visually, in patients with culture-confirmed NTM infections, the left side of figure 2c (e.g. Prevotella sp. and Veillonella sp.) matters more in acute inflammation than whether mycobacteria are above or below the limit of detection. This finding suggests a new layer of complexity to NTM pathogenesis, namely the complex interactions between the pathogen, the host and lung microbiota. The current authors have previously reported a plausible example of how non-mycobacterial lung bacteria may modulate the host immune response, influencing susceptibility to M. tuberculosis infection [25]. A likely contributing factor is pharyngeal aspiration and reflux, which surely alter lung microbiota [1], contribute to lung immunity and injury [26], and are correlated with susceptibility to tuberculosis [27] and NTM lung disease [28]. 
Since the days of the rivalry between Pasteur and Koch (who respectively discovered S. pneumoniae and M. tuberculosis a year apart in 1881 and 1882), we have known that mycobacterial infections differ microbiologically from those of typical bacterial pathogens. In the subsequent 130 years, we have learned that they also differ immunologically and therapeutically. With the current study, Sulaiman et al. [4] have revealed how profoundly they differ ecologically as well.

Conflict of interest: None declared.

Support statement: This work was supported by US National Heart, Lung and Blood Institute grants K23HL130641 and R21AI137669. Funding information for this article has been deposited with the Crossref Funder Registry.

\section{References}

1 Dickson RP, Erb-Downward JR, Huffnagle GB. Towards an ecology of the lung: new conceptual models of pulmonary microbiology and pneumonia pathogenesis. Lancet Respir Med 2014; 2: 238-246.

2 Dickson RP, Erb-Downward JR, Prescott HC, et al. Analysis of culture-dependent versus culture-independent techniques for identification of bacteria in clinically obtained bronchoalveolar lavage fluid. J Clin Microbiol 2014; 52: 3605-3613.

3 Kelly BJ, Imai I, Bittinger $\mathrm{K}$, et al. Composition and dynamics of the respiratory tract microbiome in intubated patients. Microbiome 2016; 4: 7.

4 Sulaiman I, Wu BG, Li Y, et al. Evaluation of the airway microbiome in nontuberculous mycobacteria. Eur Respir J 2018; 52: 1800810 .

5 Kendall BA, Winthrop KL. Update on the epidemiology of pulmonary nontuberculous mycobacterial infections. Semin Respir Crit Care Med 2013; 34: 87-94.

6 Morris Conway A, Gadsby N, McKenna JP, et al. 16S pan-bacterial PCR can accurately identify patients with ventilator-associated pneumonia. Thorax 2017; 72: 1046-1048.

7 Ji YE, Colston MJ, Cox RA. The ribosomal RNA ( $\mathrm{rn}$ ) operons of fast-growing mycobacteria: primary and secondary structures and their relation to $\mathrm{rrn}$ operons of pathogenic slow-growers. Microbiology 1994; 140: 2829-2840.

8 Caverly LJ, Carmody LA, Haig SJ, et al. Culture-independent identification of nontuberculous mycobacteria in cystic fibrosis respiratory samples. PLoS One 2016; 11: e0153876.

9 Kauppinen J, Mantyjarvi R, Katila ML. Mycobacterium malmoense-specific nested PCR based on a conserved sequence detected in random amplified polymorphic DNA fingerprints. J Clin Microbiol 1999; 37: 1454-1458.

10 Salter SJ, Cox MJ, Turek EM, et al. Reagent and laboratory contamination can critically impact sequence-based microbiome analyses. BMC Biol 2014; $12: 87$.

11 Marsh RL, Nelson MT, Pope CE, et al. How low can we go? The implications of low bacterial load in respiratory microbiota studies. Pneumonia 2018; $10: 7$.

12 Sibley CD, Grinwis ME, Field TR, et al. Culture enriched molecular profiling of the cystic fibrosis airway microbiome. PLoS One 2011; 6: e22702.

13 Lau JT, Whelan FJ, Herath I, et al. Capturing the diversity of the human gut microbiota through culture-enriched molecular profiling. Genome Med 2016; 8: 72.

14 Charalampous T, Richardson H, Kay GL, et al. Rapid diagnosis of lower respiratory infection using nanopore-based clinical metagenomics. bioRxiv 2018; https://doi.org/10.1101/387548.

15 Pendleton KM, Erb-Downward JR, Bao Y, et al. Rapid pathogen identification in bacterial pneumonia using real-time metagenomics. Am J Respir Crit Care Med 2017; 196: 1610-1612.

16 Pendleton KM, Erb-Downward JR, Bao Y, et al. Reply: Clinical metagenomics for the diagnosis of hospital-acquired infections: promises and hurdles. Am J Respir Crit Care Med 2017; 196: 1618-1619.

17 Dickson RP, Erb-Downward JR, Freeman CM, et al. Bacterial topography of the healthy human lower respiratory tract. mBio 2017; 8: e02287-16.

18 Gunther A, Siebert C, Schmidt R, et al. Surfactant alterations in severe pneumonia, acute respiratory distress syndrome, and cardiogenic lung edema. Am J Respir Crit Care Med 1996; 153: 176-184.

19 Meduri GU, Kanangat S, Stefan J, et al. Cytokines IL-1 $\beta$, IL-6, and TNF- $\alpha$ enhance in vitro growth of bacteria. Am J Respir Crit Care Med 1999; 160: 961-967.

20 Freestone PP, Hirst RA, Sandrini SM, et al. Pseudomonas aeruginosa-catecholamine inotrope interactions: a contributory factor in the development of ventilator-associated pneumonia? Chest 2012; 142: 1200-1210.

21 Dickson RP, Erb-Downward JR, Prescott HC, et al. Intraalveolar catecholamines and the human lung microbiome. Am J Respir Crit Care Med 2015; 192: 257-259.

22 Dickson RP, Erb-Downward JR, Huffnagle GB. Homeostasis and its disruption in the lung microbiome. Am J Physiol Lung Cell Mol Physiol 2015; 309: L1047-L1055.

23 Cambier CJ, Falkow S, Ramakrishnan L. Host evasion and exploitation schemes of Mycobacterium tuberculosis. Cell 2014; 159: 1497-1509.

24 Danelishvilli L, Bermudez LE. Role of type I cytokines in host defense against Mycobacterium avium infection. Curr Pharm Des 2003; 9: 61-65.

25 Segal LN, Clemente JC, Li Y, et al. Anaerobic bacterial fermentation products increase tuberculosis risk in antiretroviral-drug-treated HIV patients. Cell Host Microbe 2017; 21: 530-537.

26 Starosta V, Kitz R, Hartl D, et al. Bronchoalveolar pepsin, bile acids, oxidation, and inflammation in children with gastroesophageal reflux disease. Chest 2007; 132: 1557-1564.

27 Fan WC, Ou SM, Feng JY, et al. Increased risk of pulmonary tuberculosis in patients with gastroesophageal reflux disease. Int J Tuberc Lung Dis 2016; 20: 265-270.

28 Koh WJ, Lee JH, Kwon YS, et al. Prevalence of gastroesophageal reflux disease in patients with nontuberculous mycobacterial lung disease. Chest 2007; 131: 1825-1830. 